\title{
AXIALLY-SYMMETRIC BOUNDARY-VALUE PROBLEMS ${ }^{1}$
}

\author{
ALBERT E. HEINS
}

I. Introduction. The axially-symmetric boundary-value problems which we shall discuss are solutions of the elliptic partial differential equations

$$
\nabla^{2} \Phi \pm k^{2} \Phi=0
$$

where $\Phi$ is a function of the conventional cylindrical coordinates $(r, \phi, z)$ and the boundary conditions are supplied on some surface of revolution $S$. The term axially-symmetric does not refer to the fact that $\Phi(r, \phi, z)$ is independent of the angular coordinate $\phi$, but to the fact that $S$ is a surface of revolution about the $z$ axis. This address will be concerned with a class of boundary-value problems for equation (1.1) for which we supply Dirichlet, Neumann or mixedtype ${ }^{2}$ boundary data on $S$.

A classical method for studying such boundary-value problems has been the use of expansion or integral transform theorems appropriate to the coordinate system of the surface $S$, at least when they are available. Yet it is has been of ten observed that there are cases in which the special functions of the coordinate system disappear and we may be left with simple results. For the axially-symmetric geometries we consider here, this phenomenon is not accidental, and indeed, we find that the boundary-value problems of which we speak can be expressed in terms of boundary information of a two-dimensional harmonic function. This information is contained in the Poisson integral representation of solutions of the equations (1.1) [23], [24]. ${ }^{3}$ Once we supply the boundary data for equation (1.1), we have an integral equation for the two-dimensional harmonic function of which we spoke. In conjunction with the Poisson integral representation, we also have a classical one due to Helmholtz which has a Green's function associated with (1.1) as a kernel. We shall see that the continuation of axis data ${ }^{4}$ provided by the Helmholtz representa-

An address delivered before the Evanston meeting of the Society on November 23, 1962 by invitation of the Committee to Select Hour Speakers for Western Sectional Meetings; received by the editors July $5,1965$.

1 Publication supported by the Air Force Office of Scientific Research.

2 By mixed-type boundary data we mean that a linear combination of $\Phi$ and its normal derivative is assigned on $S$.

3 Numbers in brackets refer to the references cited at the end of the paper.

- That is, data supplied on the axis of symmetry $r=0$. 
tion into the complex domain produces a second integral equation which differs from the usual ones encountered in such boundary-value problems. We have here then another way of viewing these boundaryvalue problems, since now the analytically continued Helmholtz data may be combined with the Poisson data. In some cases, as we shall see, the Poisson data are adequate to solve the boundary-value problem completely, while in others we shall require both representations. Whether we shall require one or both of these depends on how much information the Poisson integral provides. We shall elaborate upon this point in the examples which we shall discuss later in this talk.

It might be added here that representations have been found for these axially-symmetric boundary-value problems which are a combination of the Poisson representation and a portion of the analytically continued Helmholtz representation. Such a result is to be found in E. Beltrami's [2] study of axially-symmetric potentials well before the turn of the last century. It was found that these potentials have Hankel transformation representations. The combined form of which we spoke arose from casting these representations into another form. The hypotheses needed to justify Beltrami's methods are much more than we actually require. A representation equivalent to that of Beltrami was found by E. R. Love [22] in 1949 by much the same procedure, that is, through the realm of special functions. Special examples of the type of integral equations which we shall derive are to be found in Beltrami's paper. Similar ones have been found by E. T. Copson [14] and D. S. Jones [21] directly from the Helmholtz representation. In connection with the axially-symmetric wave equation, W. D. Collins [8] used an expression given by H. Bateman [1] which is a generalization of the Poisson representation [18]. Upon combining it with the Helmholtz representation for the wave function due to a ring of sources, Collins produced once again a combined form. He has employed this form in a number of studies involving the axially-symmetric potential and wave equations and has often preferred to introduce special coordinates immediately, thereby tending to obscure the simplicity of the ideas involved. Noble attempts have been made to view the combined form and the component form as two different results. This, in the light of Collin's derivation, is not the case. Here we choose to defer to the separate representations of Poisson and Helmholtz because of their inherent simplicity.

It is to be recognized that we provide another formulation for this class of boundary-value problems, although whether we can solve the resulting integral equations is another question. We do achieve 
a unity of formulation and we are led to linear integral equations which are locally of the Volterra type. For such integral equations we can usually extract some information about the nature of the solution. Although the final setting is not coordinate free, we are spared the cumbersome details of the attendant analysis of special functions. There are situations in which axially-symmetric boundaryvalue problems are formulated as linear Fredholm integral equations of the first kind with the aid of the Helmholtz representation. The methods we shall discuss here convert these into a more tractable form, and even though we may not be able to solve the resulting integral equation explicitly, the Neumann iteration process turns out to be a useful tool under appropriate circumstances. The above remarks are particularly useful when $k^{2}=0$ in (1.1). If $k^{2} \neq 0$, we appear to be limited to the geometry of the circular disk at present.

II. The Poisson integral representation for Laplace's equations. We shall now give a brief account of the Poisson representation for Laplace's equation. Suppose we know that an axially-symmetric potential function is analytic and real on the $z$ axis, where by the term analytic we mean that it may be continued into some portion of the $z+i r$ plane. The Poisson representation [23] then enables us to determine the potential in the real $z-r$ plane, that is, the meridian plane of a body of revolution with respect to the $z$ axis. Indeed if $\Phi_{0}(r, z)$ is such a function, it satisfies the partial differential equation

$$
\frac{\partial^{2} \Phi_{0}}{\partial r^{2}}+\frac{1}{r} \frac{\partial \Phi_{0}}{\partial r}+\frac{\partial^{2} \Phi_{0}}{\partial z^{2}}=0
$$

and $\Phi_{0}(r, z)$ is given by the Poisson representation

$$
\Phi_{0}(r, z)=\frac{1}{\pi} \int_{0}^{\pi} \Phi_{0}(0, z+i r \cos \psi) d \psi .
$$

This representation may be written in several different forms, such as

$$
\begin{aligned}
\Phi_{0}(r, z) & =\frac{1}{i \pi} \int_{z-i r}^{z+i r} \frac{\Phi_{0}(0, t) d t}{\left[(t-z)^{2}+r^{2}\right]^{1 / 2}} \\
& =\frac{2}{\pi} \int_{0}^{r} \frac{\operatorname{Re}\left[\Phi_{0}(0, z+i t)\right] d t}{\left(r^{2}-t^{2}\right)^{1 / 2}} .
\end{aligned}
$$

(2.2) and (2.3) are useful for boundary-value problems involving Dirichlet data on a surface $S$, that is $z=f(r)$ or $r=g_{1}(s), z=g_{2}(s)$ where $s$ is the arc-length parameter of a curve in the meridian plane of $S$. 
We now turn to the case which requires the solution of equation (2.1) subject to a Neumann boundary condition on some surface $S$ of revolution. If we put $s$ as the arc-length parameter of a curve in the meridian plane of $S$, the normal derivative on this surface (up to an algebraic sign) is

$$
\begin{aligned}
& \frac{d z}{d s} \frac{\partial \Phi(r, z)}{\partial r}-\frac{d r}{d s} \frac{\partial \Phi(r, z)}{\partial z} \\
& =\frac{1}{\pi} \int_{0}^{\pi}\left[\frac{d z}{d s} \frac{\partial \Phi(0, z+i r \cos \psi)}{\partial r}-\frac{d r}{d s} \frac{\partial \Phi(0, z+i r \cos \psi)}{\partial z}\right] d \psi
\end{aligned}
$$

where now $z$ and $r$ are considered as functions of $s$ after the partial differentiations with respect to $r$ and $z$ are performed. (2.4) simplifies to

$$
\begin{aligned}
& =-\frac{i}{\pi} \int_{z-i r}^{z+i r} \frac{d t}{\left[(t-z)^{2}+r^{2}\right]^{1 / 2}} \frac{\partial \Phi(0, t)}{\partial t}\left[\frac{t-z}{r} \frac{d z}{d s}-\frac{d r}{d s}\right] \\
& =\frac{i}{\pi r} \frac{\partial}{\partial s} \int_{z-i r}^{z+i r}\left[(t-z)^{2}+r^{2}\right]^{1 / 2} \frac{\partial \Phi(0, t)}{\partial t} d t \\
& =\frac{-i}{\pi r} \frac{\partial}{\partial s} \int_{z-i r}^{z+i r} \frac{\Phi(0, t)(t-z) d t}{\left[(t-z)^{2}+r^{2}\right]^{1 / 2}} .
\end{aligned}
$$

Let us suppose that $\Phi$ is dependent on an angle $\phi$ measured with respect to some fixed meridian plane of $S$. In this case we have

$$
\frac{\partial^{2} \Phi}{\partial r^{2}}+\frac{1}{r} \frac{\partial \Phi}{\partial r}+\frac{1}{r^{2}} \frac{\partial^{2} \Phi}{\partial \phi^{2}}+\frac{\partial^{2} \Phi}{\partial z^{2}}=0,
$$

and we may expand $\Phi(r, \phi, z)$ in a Fourier series in $\phi[16]$, that is,

$$
\begin{aligned}
\Phi(r, \phi, z) & =\frac{1}{2 \pi} \int_{0}^{2 \pi} \Phi\left(r, \phi^{\prime}, z\right) d \phi^{\prime}+\frac{1}{\pi} \int_{0}^{2 \pi} \Phi\left(r, \phi^{\prime}, z\right) \cos n\left(\phi-\phi^{\prime}\right) d \phi^{\prime} \\
& =\Phi_{0}(r, z)+\sum_{n=1}^{\infty} \Phi_{n}^{0}(r, z) \cos n \phi+\sum_{n=1}^{\infty} \Phi_{n}^{*}(r, z) \sin n \phi .
\end{aligned}
$$

Here

$$
\Phi_{n}^{c}(r, z)=\frac{1}{\pi} \int_{0}^{2 \pi} \Phi\left(r, \phi^{\prime}, z\right) \cos n \phi^{\prime} d \phi^{\prime}, \quad n \geqq 1
$$

and

$$
\Phi_{n}^{\prime}(r, z)=\frac{1}{\pi} \int_{0}^{2 \pi} \Phi\left(r, \phi^{\prime}, z\right) \sin n \phi^{\prime} d \phi^{\prime}, \quad n \geqq 1,
$$


and $\Phi_{n}^{e}$ and $\Phi_{n}^{e}$ satisfy the equation

$$
\frac{\partial^{2} \Phi_{n}}{\partial r^{2}}+\frac{1}{r} \frac{\partial \Phi_{n}}{\partial r}+\frac{\partial^{2} \Phi_{n}}{\partial z^{2}}-\frac{n^{2} \Phi_{n}}{r^{2}}=0 .
$$

We introduce the change of variables $r^{n} U_{n}(r, z)=\Phi_{n}(r, z)$, and (2.6) becomes

$$
\frac{\partial^{2} U_{n}}{\partial r^{2}}+\frac{(2 n+1)}{r} \frac{\partial U_{n}}{\partial r}+\frac{\partial^{2} U_{n}}{\partial z^{2}}=0, \quad n=0,1, \cdots
$$

Now the Poisson representation [19] reads

$$
\begin{aligned}
U_{n}(r, z) & =\frac{\Gamma(n+1)}{\Gamma\left(\frac{1}{2}\right) \Gamma\left(n+\frac{1}{2}\right)} \int_{0}^{\pi} U_{n}(0, z+i r \cos \psi) \sin ^{2 n} \psi d \psi \\
& =\frac{\Gamma(n+1)}{\Gamma\left(\frac{1}{2}\right) \Gamma\left(n+\frac{1}{2}\right)} \int_{-r}^{r} U_{n}(0, z+i t) \frac{\left(r^{2}-t^{2}\right)^{n-1 / 2}}{r^{2 n}} d t \\
& =\frac{2 \Gamma(n+1)}{\Gamma\left(\frac{1}{2}\right) \Gamma\left(n+\frac{1}{2}\right)} \int_{0}^{r} \frac{\left(r^{2}-t^{2}\right)^{n-1 / 2}}{r^{2 n}} \operatorname{Re} U_{n}(0, z+i t) d t .
\end{aligned}
$$

Let us note that $n$th radial derivative of $\Phi_{n}(r, z)$ is essentially $U_{n}(0, z)$ at $r=0$ and further that $U_{n}(r, z)$ can be determined from a knowledge of the two-dimensional harmonic function $\operatorname{Re} U_{n}(0, z+i t)$. Hence, Dirichlet data on $S$ which depends on the angle $\phi$ is converted into a series of problems for which we have, in theory, $\operatorname{Re} U_{n}(0, z+i t)$, a two-dimensional harmonic function, and similar results may be derived for the Neumann problem.

It is possible to introduce an auxiliary function $V_{p}(r, z)$ which will enable us to reduce the Neumann problem for $U_{n}(r, z)$ to a Dirichlet problem for $V_{p}(r, z)$. To this end, we introduce the Beltrami-Stokes equations

$$
r^{p} \frac{\partial U_{p}}{\partial z}=\frac{\partial V_{p}}{\partial r}
$$

and

$$
\boldsymbol{r}^{p} \frac{\partial U_{p}}{\partial r}=-\frac{\partial V_{p}}{\partial z}
$$

$U_{p}$ and $V_{p}$ satisfy the partial differential equations

$$
\frac{\partial^{2} U_{p}}{\partial r^{2}}+\frac{p}{r} \frac{\partial U_{p}}{\partial r}+\frac{\partial^{2} U_{p}}{\partial z^{2}}=0
$$


and

$$
\frac{\partial^{2} V_{p}}{\partial r^{2}}-\frac{p}{r} \frac{\partial V_{p}}{\partial r}+\frac{\partial^{2} V_{p}}{\partial z^{2}}=0 .
$$

Hence with $p=2 n+1, \Phi_{(p-1) / 2}(r, z)=r^{(p-1) / 2} U_{(p-1) / 2}(r, z)$. For $p=1$, for example, the normal derivative of $\Phi_{0}(r, z)$ on a surface $S$ may be expressed in terms of $V_{1}(r, z)$ and is (up to an algebraic sign)

$$
-\frac{1}{r} \frac{\partial V_{1}}{\partial z} \frac{d z}{d s}-\frac{1}{r} \frac{d r}{d s} \frac{\partial V_{1}}{\partial r}=-\frac{1}{r} \frac{\partial V_{1}}{\partial s} .
$$

From this if follows that

$$
V_{1}(r, z)=-\frac{i}{\pi} \int_{z-i r}^{z+i r} \frac{\Phi(0, t)(t-z) d t}{\left[(t-z)^{2}+r^{2}\right]^{1 / 2}}+c
$$

on the surface $S$. The constant $c$ is arbitrary. Similar representations may be given for other odd values of $p$.

Representations of the form (2.2) were derived by Poisson [23] in 1823 in connection with the hyperbolic partial differential equation

$$
\frac{\partial^{2} U}{\partial t^{2}}=\frac{\partial^{2} U}{\partial x^{2}}+\frac{\lambda}{x} \frac{\partial U}{\partial X}+\frac{\mu U}{x^{2}}
$$

for $x$ and $t$ real variables and $\lambda$ and $\mu$ constants. His derivation would carry over without any difficulties to such equation as (2.1). The substitution $U(x, t)=x^{\alpha} v(x, t)$ reduces this equation to

$$
\frac{\partial^{2} v}{\partial t^{2}}=\frac{\partial^{2} v}{\partial x^{2}}+\frac{2 \alpha+\lambda}{x} \frac{\partial v}{\partial x}
$$

if we take $\alpha$ as a root of the quadratic equation

$$
\alpha^{2}+\alpha(\lambda-1)+\mu=0 .
$$

In characteristic variables, equation (2.9) is discussed by G. Darboux's Theorie generale des surfaces, Vol. II. In the form (2.9) it has been studied intensively since 1946 by A. Weinstein [25], [26] and his coworkers in both the elliptic and hyperbolic cases. For the elliptic version of equation (2.9) (that is, with $t$ replaced by $i t$ ) this gives rise to Weinstein's celebrated theory of the generalized axially symmetric potential, but we shall not have need of the full scope of this theory.

Upon putting $2 \alpha+\lambda=1 \pm \beta$ with $\beta=\left[(\lambda-1)^{2}-4 \mu\right]^{1 / 2}$, Poisson finds that the solutions of equation (2.9a) may be expressed as either 


$$
v(x, t)=A \int_{0}^{\pi} V_{1}(t+x \cos \omega) \sin ^{\beta} \omega d \omega
$$

or

$$
v(x, t)=B \int_{0}^{\pi} V_{2}(t+x \cos \omega) \sin ^{-\beta} \omega d \omega
$$

where $A$ and $B$ are constants and $\beta$ permits the convergence of either integral. If it is assumed that $\left[(\lambda-1)^{2}-4 \mu\right]^{1 / 2}$ is real and positive, (2.10) is convergent for $V_{1}(t)$ a continuous function of $t$ for some interval on the $t$ axis and (2.10a) is convergent for $\beta<1$ under similar circumstances. There are transformations of equation (2.9a) which enable us to satisfy these requirements. In either case if $v(0, t)$ is a continuous function of $t$ for some portion of the $t$ axis we have

$$
v(0, t)=A \int_{0}^{\pi} V_{1}(t) \sin ^{\beta} \omega d \omega
$$

or

$$
v(0, t)=B \int_{0}^{\pi} V_{2}(t) \sin ^{-\beta} \omega d \omega .
$$

Since

$$
\int_{0}^{\pi} \sin ^{\beta} \omega d \omega=\frac{\sqrt{ } \pi}{2} \frac{\Gamma((\beta+1) / 2)}{\Gamma(\beta / 2+1)}, \quad \beta>-1,
$$

we may rewrite $(2.10)$ as

$$
v(x, t)=\frac{\int_{0}^{\pi} v(0, x \cos \omega+t) \sin ^{\beta} \omega d \omega}{\int_{0}^{\pi} \sin ^{\beta} \omega d \omega}, \quad \beta>-1 .
$$

If perchance $\beta=0$, the two solutions coincide and the customary limiting process is required to find the second solution. In [23], Poisson did not write (2.10) or (2.10a) in the form (2.11). It is to be observed that subject to the above restrictions on $\beta, \partial v / \partial x=0$ at $x=0$. Since we shall only be concerned with the case $\beta \geqq 1$ in our subsequent development, we shall only use (2.11). Thus equation (2.9) and this condition define a singular Cauchy problem in the hyperbolic case, and with minor changes in notation do so also in the elliptic case [see equations (2.2) and (2.8)]. We also note that we require 
much less than the analyticity of $\Phi_{0}(0, z)$ if $(2.2)$ is to satisfy equation (2.1).

It is of historical interest to observe that Poisson found an integral representation for Bessel functions by putting $V_{1}(t+x \cos \omega)$ $=\exp (t+x \cos \omega)$. Further (2.2) is of ten referred to as the Laplace integral, although it does not seem to appear in his work. In some unpublished working notes (with no dates), T. Carleman had indicated that he was aware of some possible applications of the form (2.2) to boundary-value problems, but he does not seem to have published anything on this subject. How the usefulness of Poisson's work went unnoticed for more than a century is a mystery and major historical sources seem to give no clues.

III. Disk-like configurations. Boundary-value problems involving disks are probably amongst the most simple to which we can apply the results of the previous section. Suppose that a disk of radius unity is maintained at a constant potential $A$. Then according to the Helmholtz representation, a solution of equation (2.1) which vanishes at infinity and $\epsilon C^{2}$ (except at its rim) is given by

$$
\Phi_{0}(r, z)=\frac{1}{4 \pi} \int_{0}^{1} \int_{0}^{2 \pi}\left[\frac{\partial \Phi_{0}}{\partial n^{\prime}}\right]_{z^{\prime}=0} \frac{r^{\prime} d r^{\prime} d \phi^{\prime}}{\rho}
$$

where $\rho=\left[r^{2}+r^{\prime 2}-2 r r^{\prime} \cos \left(\phi-\phi^{\prime}\right)+z^{2}\right]^{1 / 2}$. In particular, on the axis of symmetry $r=0$.

$$
\Phi_{0}(0, z)=\frac{1}{2} \int_{0}^{1}\left[\frac{\partial \Phi_{0}}{\partial n^{\prime}}\right]_{z^{\prime}=0} \frac{r^{\prime} d r^{\prime}}{\left[r^{\prime 2}+z^{2}\right]^{1 / 2}} .
$$

On the other hand, the Poisson representation gives us

$$
A=\frac{2}{\pi} \int_{0}^{r} \frac{\operatorname{Re}\left[\Phi_{0}(0, i t)\right] d t}{\left(r^{2}-t^{2}\right)^{1 / 2}}, \quad 0<r<1 .
$$

Hence from equation (3.2) we have $\operatorname{Re}\left[\Phi_{0}(0, i t)\right], 0 \leqq t<1$, while we may use equation (3.1) to supply us with the discontinuity of the normal derivative on the disk. That is, if $z$ is continued into the domain of complex variables $z+i r$, the $\operatorname{Re}\left[\Phi_{0}(0, i r)\right]$ is simply

$$
\frac{1}{2} \int_{r}^{1}\left[\frac{\partial \Phi_{0}}{\partial n^{\prime}}\right]_{z^{\prime}=0} \frac{r^{\prime} d r^{\prime}}{\left[r^{\prime 2}-r^{2}\right]^{1 / 2}}
$$

Fortunately we have no need for the imaginary part of $\Phi_{0}(0, i r)$ which is multi-valued. We observe that equation (3.2) is an integral equation of the convolution type once we make the substitutions $r^{2}=\alpha$ 
and $t^{2}=\beta$. Hence, it can be solved and we find that $\operatorname{Re}\left[\Phi_{0}(0, i r)\right]=A$. On the other hand we learn from equation (3.3) that

$$
\frac{1}{2} \int_{r}^{1}\left[\frac{\partial \Phi_{0}}{\partial n^{\prime}}\right]_{z^{\prime}=0} \frac{r^{\prime} d r^{\prime}}{\left[r^{\prime 2}-r^{2}\right]^{1 / 2}}=A
$$

and this is also of the convolution type once the substitutions $r^{\prime 2}$ $=1-\beta$ and $r^{2}=1-\alpha$ are made. Hence we calculate quite simply the discontinuity in the normal derivative of $\Phi_{0}$ at $z^{\prime}=0$ to get

$$
\left[\frac{\partial \Phi_{0}}{\partial n^{\prime}}\right]_{z^{\prime}=0}=\frac{4}{\pi}\left(1-r^{2}\right)^{-1 / 2} \text {. }
$$

We observe that if the boundary condition on the disk depends on the angle $\phi$, we may decompose it by a Fourier decomposition and solve for each harmonic individually.

A somewhat more interesting problem deals with two parallel disks with the same axis of symmetry. In order to keep the details simple we shall assume that the disks are both of radius unity, have centers at $r=0, z=a$ and $r=0, z=-a$ and are maintained at the constant potentials $A_{1}$ and $A_{2}$ respectively. Then the Helmholtz representation gives us

$$
\Phi_{0}(0, z)=\frac{1}{2} \int_{0}^{1} \frac{B_{1}(t) t d t}{\left[(z-a)^{2}+t^{2}\right]^{1 / 2}}+\frac{1}{2} \int_{0}^{1} \frac{B_{2}(t) t d t}{\left[(z+a)^{2}+t^{2}\right]^{1 / 2}}
$$

where $B_{1}$ and $B_{2}$ are the respective discontinuities of the normal derivative of $\Phi_{0}$ on the disks in the plane $z=a$ and $z=-a$. On the other hand, the Poisson representation tells us that

and

$$
A_{1}=\frac{2}{\pi} \int_{0}^{r} \frac{\operatorname{Re}\left[\Phi_{0}(0, a+i t)\right] d t}{\left[r^{2}-t^{2}\right]^{1 / 2}}
$$

$$
A_{2}=\frac{2}{\pi} \int_{0}^{r} \frac{\operatorname{Re}\left[\Phi_{0}(0,-a+i t)\right] d t}{\left[r^{2}-t^{2}\right]^{1 / 2}} .
$$

Hence $\operatorname{Re}\left[\Phi_{0}(0, a+i t)\right]=A_{1}$ and $\operatorname{Re}\left[\Phi_{0}(0,-a+i t)\right]=A_{2}, 0 \leqq t<1$. But equation (3.4) can supply us with these quantities. Indeed we continue $z-a$ into the domain of complex variables and select the real part which is required by the Poisson representation. This gives us

$$
\begin{aligned}
A_{1} & =\operatorname{Re}\left[\Phi_{0}(0, a+i r)\right] \\
& =\frac{1}{2} \int_{r}^{1} \frac{B_{1}(t) t d t}{\left(t^{2}-r^{2}\right)^{1 / 2}}+\frac{1}{2} \operatorname{Re} \int_{0}^{1} \frac{B_{2}(t) t d t}{\left[(2 a+i r)^{2}+t^{2}\right]^{1 / 2}}
\end{aligned}
$$


and

$$
\begin{aligned}
A_{2} & =\operatorname{Re}\left[\Phi_{0}(0,-a+i r)\right] \\
& =\frac{\operatorname{Re}}{2} \int_{0}^{1} \frac{B_{1}(t) t d t}{\left[(i r-2 a)^{2}+t^{2}\right]^{1 / 2}}+\frac{1}{2} \int_{r}^{1} \frac{B_{2}(t) t d t}{\left[t^{2}-r^{2}\right]^{1 / 2}} .
\end{aligned}
$$

The solution follows immediately by the following device. Let

$$
D_{i}(r)=\int_{r}^{1} \frac{B_{i}(t) t d t}{\left(t^{2}-r^{2}\right)^{1 / 2}}, \quad i=1,2 .
$$

This may be inverted to give us

$$
B_{i}(r)=-\frac{2}{\pi} \int_{r}^{1}\left(t^{2}-r^{2}\right)^{-1 / 2} \frac{d D_{i}(t)}{d t} d t, \quad i=1,2,
$$

where we have assumed that $B_{i}(t)\left(t^{2}-r^{2}\right)^{-1 / 2}$ is integrable and therefore $D_{i}(1)=0$. Using these expressions for $B_{i}(t)$, we see that equations (3.5) and (3.6) become

$$
2 A_{1}=D_{1}(r)+\frac{2}{\pi} \int_{0}^{1} D_{2}(\tau)\left[\frac{a}{4 a^{2}+(r-\tau)^{2}}+\frac{a}{4 a^{2}+(r+\tau)^{2}}\right] d \tau
$$

and

$$
2 A_{2}=D_{2}(r)+\frac{2}{\pi} \int_{0}^{1} D_{1}(\tau)\left[\frac{a}{4 a^{2}+(r-\tau)^{2}}+\frac{a}{4 a^{2}+(r+\tau)^{2}}\right] d \tau
$$

The question of boundary conditions which depend on the radial distance $r$ may be dealt with similarly. Indeed, if the boundary conditions on the disk depend on the meridian angle, we have described the essential machinery to formulate this problem. The radii of the disks may also be unequal or there may be more than two disks and the present apparatus carries over. Clearly, since $a>0,(3.6)$ and (3.7) are regular Fredholm integral equations of the second kind and may be converted to similar integral equations for $D_{1}+D_{2}$ and $D_{1}-D_{2}$. We may therefore study the properties of the solution of these integral equations with the facts known about them. Let us observe that we neither required special functions nor expansion theorems to formulate this problem, the latter having been absorbed into the Poisson representation.

As a final example of problems involving the disk, we consider one of radius unity in the plane $z=0$ with center at the origin and which is between and parallel to two infinite planes. These planes are de- 
scribed geometrically by the coordinates $z=a$ and $-a$. Suppose, they are maintained at potential zero and the disk is maintained at potential $f(r), 0 \leqq r \leqq 1$. The obvious step is to write a Helmholtz representation for this problem in terms of the Green's function for the parallel plate region and to require that the Green's function vanishes at $z=a$ and $-a$. Then an application of Green's theorem with the assumption that the potential function vanishes sufficiently rapidly at infinity gives us a representation for $\Phi(r, z)$ on the axis of symmetry $r=0$ which obeys the boundary conditions we gave on the parallel plates. That is, $\Phi_{0}(0, z)=\frac{1}{2} \int_{0}^{1} G(0, z, t, 0) t B(t) d t$ and $B(t)$ is the discontinuity of the normal derivative of $\Phi$ on the disk. Now we have two choices in describing $G\left(r, z, t, z^{\prime}\right)$. It may be given by an integral representation or may be given by the image method of Kelvin. The latter form is simply

$$
G(0, z, t, 0)=\frac{1}{2} \sum_{m=-\infty}^{\infty} \frac{(-)^{m}}{\left[(2 m a-z)^{2}+t^{2}\right]^{1 / 2}} .
$$

From the Poisson representation we get

$$
f(r)=\frac{2}{\pi} \operatorname{Re} \int_{0}^{r} \frac{\Phi_{0}(0, i t) d t}{\left[r^{2}-t^{2}\right]^{1 / 2}}
$$

while the continuation of $\Phi_{0}(0, z)$ into the domain of the complex variable $z$ for purely imaginary $z=i \tau$ gives us

$$
\operatorname{Re} \Phi_{0}(0, i \tau)=\frac{\operatorname{Re}}{2} \int_{0}^{1} t B(t) \sum_{m=-\infty}^{\infty} \frac{(-)^{m}}{\left[(2 m a-i \tau)^{2}+t^{2}\right]^{1 / 2}} d t .
$$

It is not difficult to simplify this last sum. Put

$$
\int_{\tau}^{1} \frac{t B(t) d t}{\left[t^{2}-\tau^{2}\right]^{1 / 2}}=C(\tau)
$$

which is merely the real part of the term $m=0$ in the sum. Then as we have seen, we may express the sum less the term $m=0$ in terms of $C(\tau)$, to get

$$
\begin{aligned}
& \operatorname{Re} \Phi_{0}(0, i \tau)=\frac{1}{2} C(\tau) \\
&+\frac{1}{\pi} \int_{0}^{1} C(t) d t \sum_{m=1}^{\infty}\left[\frac{(-)^{m} 2 m}{4 m^{2}+(\tau-t)^{2}}+\frac{(-)^{m} 2 m}{4 m^{2}+(\tau+t)^{2}}\right] \\
& 0<\tau<1 .
\end{aligned}
$$


The left side of this equation is determined by the solution of the Abel-type integral equation (3.9). Had the potential been given on the planes $z=a$ and $z=-a$, two further known terms would appear in terms of the $z^{\prime}$ derivative of $G\left(r, z, t, z^{\prime}\right)$ on these planes and hence another pair of inhomogeneous terms would be added. Of course, there is an integrability requirement on these boundary terms which is not difficult to describe.

We observe that equation (3.10) can be cast into the form given by Collins [6]. The present derivation has the advantage that the analysis is elementary and the source of the derivation of the integral equation is given.

IV. A boundary value problem for a lens. In this section we shall discuss a Dirichlet problem for a body of revolution with a curvilinear boundary. We consider the lens which is the figure of revolution formed by rotating two intersecting circular arcs about the straight line joining their centers. This figure is conveniently described by peripolar (toroidal) coordinates, that is,

$$
\begin{aligned}
& x=c(\cosh \eta-\cos \theta)^{-1} \sinh \eta \cos \phi, \\
& y=c(\cosh \eta-\cos \theta)^{-1} \sinh \eta \sin \phi, \\
& z=c(\cosh \eta-\cos \theta)^{-1} \sin \theta,
\end{aligned}
$$

$\eta>0,-\pi \leqq \theta<\pi, 0 \leqq \phi<2 \pi$. The constant $2 c$ is the diameter of the intersecting circle of the two spherical zones. The faces of the lens can be characterized by $\theta=\alpha$ and $\theta=\beta$ where $-\pi<\alpha<\beta<\pi$. In any meridian plane, this transformation is equivalent to

$$
z+i r=c \cot [(\theta-i \eta) / 2]
$$

and hence the exterior of the lens in the $z-r$ plane is mapped by (4.1) in to the semi-infinite strip $\eta>0, \alpha \leqq \theta \leqq \beta$.

For simplicity of detail, let us seek a solution of equation (2.1) which reduces to a constant $U$ on the surfaces $\theta=\alpha$ and $\theta=\beta, \eta>0$. From the Poisson representation we have in terms of peripolar coordinates

$$
\begin{aligned}
& \Phi(r, z)=\tilde{\Phi}(\theta, \eta) \\
& =-\frac{1}{2 \pi} \int_{-\eta}^{\eta} \frac{\Phi\left[0, c \cot \left(\frac{\theta-i \sigma}{2}\right)\right] \csc \left(\frac{\theta-i \sigma}{2}\right)(\cosh \eta-\cos \theta)^{1 / 2} d \sigma}{(\cosh \eta-\cosh \sigma)^{1 / 2}} .
\end{aligned}
$$

Now since $\Phi(0, \lambda)$ is real for $\lambda$ real, this may be rewritten as 


$$
\Phi(r, z)=-\frac{1}{\pi} \int_{0}^{\eta}
$$

$$
\frac{\operatorname{Re}\left\{\Phi\left[0, c \cot \left(\frac{\theta-i \sigma}{2}\right)\right] \csc \left(\frac{\theta-i \sigma}{2}\right)\right\}(\cosh \eta-\cos \theta)^{1 / 2} d \sigma}{(\cosh \eta-\cosh \sigma)^{1 / 2}} .
$$

Hence we may use (4.2) to express the boundary data in terms of the harmonic function

$$
g(\theta, \sigma)=\operatorname{Re}\left\{\Phi\left[0, c \cot \left(\frac{\theta-i \sigma}{2}\right)\right] \csc \left(\frac{\theta-i \sigma}{2}\right)\right\}
$$

at $\theta=\alpha$ and $\beta$. Indeed, these harmonic functions appear as solutions of two Abel type integral equations. That is,

$$
U(\cosh \eta-\cos \alpha)^{-1 / 2}=-\frac{1}{\pi} \int_{0}^{\eta} g(\alpha, \sigma)(\cosh \eta-\cosh \sigma)^{-1 / 2} d \sigma
$$

and

$$
U(\cosh \eta-\cos \beta)^{-1 / 2}=-\frac{1}{\pi} \int_{0}^{\eta} g(\beta, \sigma)(\cosh \eta-\cosh \sigma)^{-1 / 2} d \sigma .
$$

These integral equations may be reduced to a more customary form by the substitutions $t+1=\cosh \sigma$ and $s+1=\cosh \eta$, and we find that

$$
g(\alpha, \eta)=-U \sin \alpha / 2 \cosh \eta / 2(\cosh \eta-\cos \alpha)^{-1}
$$

and

$$
g(\beta, \eta)=-U \sin \beta / 2 \cosh \eta / 2(\cosh \eta-\cos \beta)^{-1} \text {. }
$$

Now we have the information that $g(\alpha, \eta)$ and $g(\beta, \eta)$ are the boundary values of a two-dimensional harmonic function which is even in the variable $\eta$. Various methods are at our disposal to determine $g(\theta, \eta)$, one of them being to form the Green's function $G\left(\theta, \sigma, \theta^{\prime}, \sigma^{\prime}\right)$ for the two-dimensional Laplace equation subject to the boundary condition $G=0$ for $\theta^{\prime}=\alpha$ and $\theta^{\prime}=\beta$. With this Green's function, we can write (subject to the condition that $g(\theta, \sigma)$ vanishes sufficiently rapidly at infinity)

$$
g(\theta, \sigma)=\int_{-\infty}^{\infty}\left\{\left.g\left(\alpha, \sigma^{\prime}\right) \frac{\partial G}{\partial \theta^{\prime}}\right|_{0-\alpha}-\left.g\left(\beta, \sigma^{\prime}\right) \frac{\partial G}{\partial \theta^{\prime}}\right|_{\theta^{\prime}-\beta}\right\} d \sigma^{\prime},
$$

where 


$$
G\left(\theta, \sigma, \theta^{\prime}, \sigma^{\prime}\right)=\frac{1}{4 \pi} \ln \left\{\frac{\cosh \frac{\pi\left(\sigma-\sigma^{\prime}\right)}{\gamma}-\cos \frac{\pi}{\gamma}\left(\theta+\theta^{\prime}-2 \alpha\right)}{\cosh \frac{\pi\left(\sigma-\sigma^{\prime}\right)}{\gamma}-\cos \frac{\pi}{\gamma}\left(\theta-\theta^{\prime}\right)}\right\}
$$

and $\gamma=\beta-\alpha$. Hence, save for the details of evaluating the integrals in (4.3) we have provided a representation for the boundary-value problem we proposed. We have had, of course, good fortune here, since the Poisson integral has provided all of the information which we needed. This situation does not always prevail. For example, if instead of two intersecting circular arcs, we had nonintersecting ones, there would not be enough information provided by the Poisson representation. Indeed, in this latter situation, $g(\alpha, \sigma)$ and $g(\beta, \sigma)$ could only be determined in some finite and symmetric intervals of the $\theta$ axis and this would not be adequate to determine $g(\theta, \sigma)$ in the strip. However, as we shall see presently, a knowledge of $g(\alpha, \sigma)$ and $g(\beta, \sigma)$ when used in conjunction with the Helmholtz representation provides further facts.

Since we have provided integral representation (2.5) for the normal derivative, it is clear that we may discuss the Neumann problem for the lens in a fashion similar to the one which we used to discuss the Dirichlet problem. As a consequence of the derivative in (2.5), an arbitrary constant is introduced. It may be evaluated by specifying special behaviour of $\Phi(r, z)$ as $\left(r^{2}+z^{2}\right) \rightarrow \infty$ or of $\Phi(r, z)$ in the neighborhood of the intersection of the two spherical zones, but we shall not pursue these points.

V. A boundary value problem for a lens: Alternative discussion. There is a second method which we shall now discuss and which provides another integral equation. In $§ I V$, the Poisson representation determined a harmonic function in two variables from boundaryvalue data in three. Now we shall find an integral equation which can be solved and is derived from the simple layer representation of potential theory. Such a representation, which is independent of $\phi$ and vanishes at infinity, is given by

$$
\Phi(0, z)=\frac{1}{2 \pi} \iint \frac{\Lambda(P) d A}{\left[x_{1}^{2}+y_{1}^{2}+\left(z-z_{1}\right)^{2}\right]^{1 / 2}},
$$

where the surface integral is evaluated over both faces on the lens. In view of the independence of $\Phi$ and therefore $\Lambda$ on the angle $\phi$, (5.1) may be written as an integral in a meridian plane as 


$$
\Phi(0, z)=\int \frac{\Lambda(P) d s}{\left[x_{1}^{2}+y_{1}^{2}+\left(z-z_{1}\right)^{2}\right]^{1 / 2}}
$$

where $s$ is the arc-length parameter of the trace of the lens in the meridian plane. Since each face of the lens may be characterized by the peripolar coordinates $\theta=\alpha$ and $\theta=\beta,(5.2)$ may be written explicity as

$$
\begin{aligned}
\Phi(0, z) & =\int_{0}^{\infty} \frac{\Lambda_{\alpha}\left(\eta^{\prime}\right) d \eta^{\prime}}{\left\{\left[z-c \cot \left(\frac{\alpha-i \eta^{\prime}}{2}\right)\right]\left[z-c \cot \left(\frac{\alpha+i \eta^{\prime}}{2}\right)\right]\right\}^{1 / 2}} \\
& +\int_{0}^{\infty} \frac{\Lambda_{\beta}\left(\eta^{\prime}\right) d \eta^{\prime}}{\left\{\left[z-c \cot \left(\frac{\beta-i \eta^{\prime}}{2}\right)\right]\left[z-c \cot \left(\frac{\beta+i \eta^{\prime}}{2}\right)\right]\right\}^{1 / 2}} .
\end{aligned}
$$

$\Lambda_{\alpha}\left(\eta^{\prime}\right)$ and $\Lambda_{\beta}\left(\eta^{\prime}\right)$ are the simple layer distributions on the respective surfaces $\theta^{\prime}=\alpha$ and $\theta^{\prime}=\beta$ multiplied by an appropriate factor to permit integration with respect to $\eta^{\prime}$.

Now we derive the integral equation of which we spoke by continuing the variable $z$ into the complex domain by the transformation $z=c \cot ((\alpha-i \sigma) / 2)$. This will give us $\Phi[0, c \cot ((\alpha-i \sigma) / 2)]$, and its real part has already been provided by the Poisson representation in $\S I V$. Hence, equation (5.3) becomes

$$
\begin{aligned}
\Phi\left[0, c \cot \left(\frac{\alpha-i \sigma}{2}\right)\right] \csc \left(\frac{\alpha-i \sigma}{2}\right) \\
=\frac{1}{c} \int_{0}^{\infty} \frac{\Lambda_{\beta}(\eta)(\cosh \eta-\cos \beta)^{1 / 2} d \eta}{[\cosh \eta-\cos (\beta-\alpha+i \sigma)]^{1 / 2}} \\
+\frac{1}{c} \int_{0}^{\infty} \frac{\Lambda_{\alpha}(\eta)(\cosh \eta-\cos \alpha)^{1 / 2} d \eta}{[\cosh \eta-\cosh \sigma]^{1 / 2}} .
\end{aligned}
$$

We might have some concern about the choice of the branch of $(\cosh \eta-\cosh \sigma)^{1 / 2}$ when $0<\eta<\sigma$, but we observe that all we have in (5.4), is the real part of the left side. Hence

$$
\begin{aligned}
\operatorname{Re}\left\{\Phi\left[0, c \cot \left(\frac{\alpha-i \sigma}{2}\right)\right] \csc \left(\frac{\alpha-i \sigma}{2}\right)\right\} \\
=\frac{1}{c} \operatorname{Re} \int_{0}^{\infty} \frac{\Lambda_{\beta}(\eta)(\cosh \eta-\cos \beta)^{1 / 2} d \eta}{[\cosh \eta-\cos (\beta-\alpha+i \sigma)]^{1 / 2}} \\
\quad+\frac{1}{c} \int_{0}^{\infty} \frac{\Lambda_{\alpha}(\eta)(\cosh \eta-\cos \alpha)^{1 / 2} d \eta}{(\cosh \eta-\cosh \sigma)^{1 / 2}}, 0 \leqq \sigma .
\end{aligned}
$$


There is a second such equation found by interchanging $\alpha$ and $\beta$, and in both cases the left-hand side of the equation is known thanks to the information we have found in §IV.

Following Collins [3], we may cast equation (5.5) into a more familiar form. Let

$$
m_{\alpha}(\sigma)=\int_{0}^{\infty} \frac{\Lambda_{\alpha}(\eta)(\cosh \eta-\cos \alpha)^{1 / 2} d \eta}{(\cosh \eta-\cosh \sigma)^{1 / 2}},
$$

and

$$
m_{\beta}(\sigma)=\int_{0}^{\infty} \frac{\Lambda_{\beta}(\eta)(\cosh \eta-\cos \beta)^{1 / 2} d \eta}{(\cosh \eta-\cosh \sigma)^{1 / 2}}
$$

Each of these expressions may be inverted and we obtain $(\cosh \eta-\cos \alpha)^{1 / 2} \Lambda_{\alpha}(\eta)$

$$
\begin{aligned}
= & \frac{-(\cosh \eta)^{1 / 2}}{\pi} \frac{d}{d \eta} \\
& (\cosh \eta)^{1 / 2} \int_{\eta}^{\infty} \frac{m_{\alpha}(\sigma) \tanh \sigma d \sigma}{(\cosh \sigma-\cosh \eta)^{1 / 2}} \\
= & -\frac{1}{\pi} \int_{\eta}^{\infty} \frac{\sinh \eta}{(\cosh \sigma-\cosh \eta)^{1 / 2}} \frac{d m_{\alpha}}{d \sigma} d \sigma
\end{aligned}
$$

and

$$
(\cosh \eta-\cos \beta)^{1 / 2} \Lambda_{\beta}(\eta)=-\frac{1}{\pi} \int_{\eta}^{\infty} \frac{\sinh \eta}{(\cosh \sigma-\cosh \eta)^{1 / 2}} \frac{d m_{\beta}}{d \sigma} d \sigma .
$$

Equation (5.5) may then be rewritten as

$$
\begin{aligned}
c \operatorname{Re}\left\{\Phi\left[0, c \cot \left(\frac{\alpha-i \sigma}{2}\right)\right] \csc \left(\frac{\alpha-i \sigma}{2}\right)\right\} \\
=m_{\alpha}(\sigma)-\operatorname{Re} \int_{0}^{\infty} \frac{d \eta^{\prime}}{\left[\cosh \eta^{\prime}-\cos (\beta-\alpha+i \sigma)\right]^{1 / 2}} \\
\qquad \int_{\eta^{\prime}}^{\infty} \frac{\sinh \eta^{\prime}}{\left[\cosh \eta^{\prime \prime}-\cosh \eta^{\prime}\right]^{1 / 2}} \frac{d m_{\beta}}{d \eta^{\prime \prime}} d \eta^{\prime \prime}
\end{aligned}
$$

The integral may be simplified by interchanging the order of integration and integrating by parts to give 


$$
\begin{aligned}
c \operatorname{Re}\left\{\Phi\left[0, c \cot \left(\frac{\alpha-i \eta}{2}\right)\right] \csc \left(\frac{\alpha-i \eta}{2}\right)\right\} \\
=m_{\alpha}(\eta)+\operatorname{Re} \int_{0}^{\infty} \frac{m_{\beta}\left(\eta^{\prime}\right) \cosh \frac{\eta^{\prime}}{2} \sin \left(\frac{\beta-\alpha+i \eta^{\prime}}{2}\right) d \eta^{\prime}}{\cosh \eta^{\prime}-\cos (\beta-\alpha+i \eta)} \\
=m_{\alpha}(\eta)+\frac{1}{\pi} \int_{0}^{\infty} \frac{m_{\beta}\left(\eta^{\prime}\right) \sin \left(\frac{\beta-\alpha}{2}\right) \cosh \left(\frac{\eta^{\prime}-\eta}{2}\right) d \eta^{\prime}}{\cosh \left(\eta^{\prime}-\eta\right)-\cos (\beta-\alpha)} \\
+\frac{1}{\pi} \int_{0}^{\infty} \frac{m_{\beta}\left(\eta^{\prime}\right) \sin \left(\frac{\beta-\alpha}{2}\right) \cosh \left(\frac{\eta^{\prime}+\eta}{2}\right) d \eta^{\prime}}{\cosh \left(\eta^{\prime}+\eta\right)-\cos (\beta-\alpha)} .
\end{aligned}
$$

The symmetry properties of $\Lambda_{\alpha}(\eta)$ and $\Lambda_{\beta}(\eta)$ permit us to combine the last two integrals, and we have finally

$$
\begin{gathered}
c \operatorname{Re}\left\{\Phi\left[0, c \cot \left(\frac{\alpha-i \eta}{2}\right)\right] \csc \left(\frac{\alpha-i \eta}{2}\right)\right\} \\
=m_{\alpha}(\eta)+\frac{1}{\pi} \int_{-\infty}^{\infty} \frac{m_{\beta}\left(\eta^{\prime}\right) \sin \left(\frac{\beta-\alpha}{2}\right) \cosh \left(\frac{\eta^{\prime}-\eta}{2}\right) d \eta^{\prime}}{\cosh \left(\eta^{\prime}-\eta\right)-\cos (\beta-\alpha)} .
\end{gathered}
$$

There is a second integral equation of this form in which the $\alpha$ and $\beta$ are interchanged. These are standard type convolution integral equations of the Picard type and their solution has been discussed by Collins in [3]. A comparison of the formation of equation (5.6) by the present methods with those employed in [3] might be of some interest.

VI. The wave equation. The Poisson integral representation (2.2) may be generalized to satisfy the axially-symmetric wave equation

$$
\frac{\partial^{2} \Phi}{\partial y^{2}}+\frac{1}{y} \frac{\partial \Phi}{\partial y}+\frac{\partial^{2} \Phi}{\partial z^{2}} \pm k^{2} \Phi=0 .
$$

In this case, we have that

$$
\Phi(y, z)=\frac{1}{\pi} \int_{0}^{\pi} \Phi(0, z+i y \cos \psi) \cos (k y \sin \psi) d \psi
$$

or 


$$
\Phi(y, z)=\frac{1}{\pi} \int_{0}^{\pi} \Phi(0, z+i y \cos \psi) \cosh (k y \sin \psi) d \psi .
$$

The form (6.2) satisfies equation (6.1) with the plus sign before the $k^{2}$, while the form (6.3) satisfies the same equation with the minus sign before the $k^{2}$. We may remark at this point, that equation (6.1) may be generalized to the form

$$
\frac{\partial^{2} \Phi}{\partial y^{2}}+\frac{\lambda}{y} \frac{\partial \Phi}{\partial y}+\frac{\partial^{2} \Phi}{\partial z^{2}} \pm k^{2} \Phi=0
$$

for $\lambda$ an arbitrary and real constant. In particular if $\lambda$ is an odd and positive integer, the situation which we had in $\S I$ is available with minor changes. Indeed the first form of (2.8) is merely modified by the extra term $\cos (k y \sin \psi)$ or $\cosh (k y \sin \psi)$. There is however, a great difference in the effectiveness of such forms as (6.2) and (6.3). While some of the coordinate transformations we employed in $\S \S I I$ and III gave relatively simple integral equations to solve, the presence of the cosine or hyperbolic cosine term adds difficulties except in the case of a disk and the related configurations which we discussed in $\S \S I I$ and III.

We shall only discuss one example here, since many of the methods we can use have already been described earlier in this paper. The problem to be considered is a generalization of the first one discussed in §III and has its origin in the theory of diffraction. Let us suppose that a plane wave is normally incident from the left upon a "soft disk" of radius unity. Such a plane wave is a solution of equation (6.1) with the plus sign and has the functional form $\exp (i k z)$. The Helmholtz representation provides us with

$$
\Phi(0, z)=\exp (i k z)+\int_{0}^{1} A(t) \frac{\exp \left[i k\left(t^{2}+z^{2}\right)^{1 / 2}\right]}{\left(t^{2}+z^{2}\right)^{1 / 2}} t d t
$$

where $A(t)$ is essentially the discontinuity of the normal derivative of $\Phi$ on the disk. Had we required $\Phi$ at any point in space, the integral in (6.5) would have been taken over the area of the disk and the term $\left(t^{2}+z^{2}\right)^{1 / 2}$ would have been replaced by the distance from the point in space to a point on the disk. The axially symmetric situation which we have described permits the simple form (6.5) on the axis.

Now we shall show how (6.5) with the Poisson representation (6.2) produces another integral equation. Since (6.2) requires $\Phi(0, i y+0)$ and $\Phi(0,0-i y)$, that is

$$
\operatorname{Lim}_{\epsilon \rightarrow 0} \Phi(0, \epsilon+i y) \text { and } \operatorname{Lim}_{\epsilon \rightarrow 0} \Phi(0, \epsilon-i y),
$$


$\epsilon>0$, we shall examine these limits first. From (6.5) we get

$$
\begin{aligned}
\Phi(0,0+i y)=\exp (-k y) & +i \int_{0}^{y} A(t) \frac{\exp \left[k\left(y^{2}-t^{2}\right)^{1 / 2}\right]}{\left(y^{2}-t^{2}\right)^{1 / 2}} t d t \\
& +\int_{y}^{1} A(t) \frac{\exp \left[i k\left(t^{2}-y^{2}\right)^{1 / 2}\right]}{\left(t^{2}-y^{2}\right)^{1 / 2}} t d t
\end{aligned}
$$

and

$$
\begin{aligned}
\Phi(0,0-i y)=\exp (k y) & -i \int_{0}^{y} A(t) \frac{\exp \left(-k\left(y^{2}-t^{2}\right)^{1 / 2}\right) t d t}{\left(y^{2}-t^{2}\right)^{1 / 2}} \\
& +\int_{y}^{1} A(t) \frac{\exp \left(i k\left(t^{2}-y^{2}\right)^{1 / 2}\right) t d t}{\left(t^{2}-y^{2}\right)^{1 / 2}} .
\end{aligned}
$$

Hence we get, upon addition of equation (6.6) and (6.7)

$$
\begin{aligned}
\Phi(0,0+i y)+\Phi(0,0-i y) & \\
=2 \cosh k y & +2 i \int_{0}^{y} A(t) \frac{\sinh \left(k\left(y^{2}-t^{2}\right)^{1 / 2}\right) t d t}{\left(y^{2}-t^{2}\right)^{1 / 2}} \\
& +2 \int_{y}^{1} A(t) \frac{\exp \left(i k\left(t^{2}-y^{2}\right)^{1 / 2}\right) t d t}{\left(t^{2}-y^{2}\right)^{1 / 2}}
\end{aligned}
$$

Since the disk is "soft," we have that $\Phi(y, 0)$ vanishes for $0 \leqq y<1$. But then the Poisson representation (6.2) tells us that $\Phi(0,0+i y)$ $+\Phi(0,0-i y)=0$. Hence (6.8) becomes

$$
\begin{aligned}
& \cosh k y+i \int_{0}^{y} A(t) \frac{\sinh \left(k\left(y^{2}-t^{2}\right)^{1 / 2}\right) t d t}{\left(y^{2}-t^{2}\right)^{1 / 2}} \\
& \quad+\int_{y}^{1} A(t) \frac{\exp \left(i k\left(t^{2}-y^{2}\right)^{1 / 2}\right) t d t}{\left(t^{2}-y^{2}\right)^{1 / 2}}=0 .
\end{aligned}
$$

A similar discussion for $\epsilon \rightarrow 0^{-}$produces equation (6.8) although there are some changes in signs in (6.6) and (6.7).

We now cast equation (6.9) into a more useable form by rewriting it as follows. Since $\sinh \left(k\left(y^{2}-t^{2}\right)^{1 / 2}\right) /\left(y^{2}-t^{2}\right)^{1 / 2}$ is single-valued, we have

$$
\cosh k y+i \int_{0}^{1} \frac{\sinh \left(k\left(y^{2}-t^{2}\right)^{1 / 2}\right)}{\left(y^{2}-t^{2}\right)^{1 / 2}} A(t) t d t
$$

$$
+\int_{y}^{1} \frac{\cos \left(k\left(t^{2}-y^{2}\right)^{1 / 2}\right)}{\left(t^{2}-y^{2}\right)^{1 / 2}} A(t) t d t=0, \quad 0 \leqq y<1 .
$$

There are two ways to convert this to a more useable form. In both methods we are guided by the remarks which we made in §III. In 
the first method we change the limits by the substitutions $1-y^{2}=\alpha$ and $1-t^{2}=\beta$. Then (6.9a) becomes

$$
\begin{aligned}
2 \cosh \left[k(1-\alpha)^{1 / 2}\right] & +i \int_{0}^{1} \frac{\sinh \left(k(\beta-\alpha)^{1 / 2}\right)}{(\beta-\alpha)^{1 / 2}} A\left[(1-\beta)^{1 / 2}\right] d \beta \\
& +\int_{0}^{\alpha} \frac{\cos k(\alpha-\beta)^{1 / 2}}{(\alpha-\beta)^{1 / 2}} A\left[(1-\beta)^{1 / 2}\right] d \beta=0 .
\end{aligned}
$$

If the second integral is inverted, we get a Fredholm integral equation of the second kind. That is

$$
\begin{aligned}
& A\left[(1-\alpha)^{1 / 2}\right] \\
& +\frac{i}{\pi} \frac{d}{d \alpha} \int_{0}^{\alpha} \frac{\cosh \left(k(\alpha-\beta)^{1 / 2}\right)}{(\alpha-\beta)^{1 / 2}} d \beta \int_{0}^{1} \frac{\sinh \left(k(\rho-\beta)^{1 / 2}\right)}{(\rho-\beta)^{1 / 2}} A\left[(1-\rho)^{1 / 2}\right] d \rho \\
& \quad+\frac{2}{\pi} \frac{d}{d \alpha} \int_{0}^{\alpha} \frac{\cosh \left(k(\alpha-\beta)^{1 / 2}\right)}{(\alpha-\beta)^{1 / 2}} \cosh \left[k(1-\beta)^{1 / 2}\right] d \beta=0 .
\end{aligned}
$$

Upon simplifying this last equation we get [15], [17]

$$
\begin{aligned}
A\left[(1-\alpha)^{1 / 2}\right]+ & \frac{i}{2 \pi(\alpha)^{1 / 2}} \int_{0}^{1}\left[\frac{\sinh k\left((\rho)^{1 / 2}+(\alpha)^{1 / 2}\right)}{(\rho)^{1 / 2}+(\alpha)^{1 / 2}}\right. \\
& \left.+\frac{\sinh k\left((\rho)^{1 / 2}-(\alpha)^{1 / 2}\right)}{(\rho)^{1 / 2}-(\alpha)^{1 / 2}}\right] A\left[(1-\rho)^{1 / 2}\right] d \rho \\
+ & \frac{2}{\pi} \frac{d}{d \alpha} \int_{0}^{\alpha} \frac{\cosh k(\alpha-\beta)^{1 / 2}}{(\alpha-\beta)^{1 / 2}} \cosh \left[k(1-\beta)^{1 / 2}\right] d \beta=0 .
\end{aligned}
$$

If we now put $B(u)=u A\left(1-u^{2}\right)^{1 / 2}$ this last equation may be rewritten as

$$
\begin{aligned}
B(u) & +\frac{i}{\pi} \int_{0}^{1} B(v)\left[\frac{\sinh k(u+v)}{u-v}+\frac{\sinh k(u-v)}{u-v}\right] d v \\
& +\frac{2}{\pi} \frac{d}{d u} \int_{0}^{u} \frac{\cosh k\left(u^{2}-v^{2}\right)^{1 / 2}}{\left(u^{2}-v^{2}\right)^{1 / 2}} \cosh \left[k\left(1-v^{2}\right)^{1 / 2}\right] v d v=0 .
\end{aligned}
$$

This is a regular Fredholm integral equation of the second kind which for $k$ sufficiently small may be solved by the Neumann iteration procedure [17].

A second reduced form may be found following a device due to Collins [3]. We put

$$
C(\alpha)=\int_{0}^{\alpha} \frac{\cos k(\alpha-\beta)^{1 / 2}}{(\alpha-\beta)^{1 / 2}} A\left[(1-\beta)^{1 / 2}\right] d \beta .
$$


Then

$$
\begin{aligned}
A\left[(1-\alpha)^{1 / 2}\right] & =\frac{1}{\pi} \frac{d}{d \alpha} \int_{0}^{\alpha} \frac{C(\beta) \cosh k(\alpha-\beta)^{1 / 2}}{(\alpha-\beta)^{1 / 2}} d \beta \\
& =\frac{1}{\pi} \int_{0}^{\alpha} \frac{d C}{d \beta} \frac{\cosh k(\alpha-\beta)^{1 / 2}}{(\alpha-\beta)^{1 / 2}} d \beta .
\end{aligned}
$$

Hence, equation (6.10) becomes

$2 \cosh \left[k(1-\alpha)^{1 / 2}\right]+C(\alpha)$

$$
+\frac{i}{\pi} \int_{0}^{1} \frac{\sinh k(\beta-\alpha)^{1 / 2}}{(\beta-\alpha)^{1 / 2}} d \beta \int_{0}^{\beta} \frac{d C}{d \gamma} \frac{\cosh k(\beta-\gamma)^{1 / 2}}{(\beta-\gamma)^{1 / 2}} d \gamma=0 .
$$

This in turn, may be reduced to

$$
\begin{aligned}
& 2 \cosh \left[k(1-\alpha)^{1 / 2}\right]+C(\alpha)+\frac{i}{2 \pi} \int_{0}^{1} \frac{C(\gamma)}{(1-\gamma)^{1 / 2}} \\
& \cdot\left[\frac{\sinh k\left((1-\alpha)^{1 / 2}+(1-\gamma)^{1 / 2}\right)}{(1-\alpha)^{1 / 2}+(1-\gamma)^{1 / 2}}+\frac{\sinh k\left((1-\alpha)^{1 / 2}-(1-\gamma)^{1 / 2}\right)}{(1-\alpha)^{1 / 2}-(1-\gamma)^{1 / 2}}\right] d \gamma=0 .
\end{aligned}
$$

Hence, we still have a regular Fredholm integral equation of the second kind and in fact practically the same equation save for the nonhomogeneous term. The second form appears to be easier to handle as far as the iteration procedure is concerned, although having found $C[\alpha]$, there is still the task of finding $A(y)$.

\section{Bibliography}

1. H. Bateman, Partial differential equations of mathematical physics, Cambridge Univ. Press, New York, 1952.

2. E. Beltrami, Sulla teoria delle funzioni potenziali simmetriche, Mem. Accad. Sci. Ist. Bologna (4) 2 (1880), 461-505.

3. W. D. Collins $O$ the solution of some axisymmetric boundary value problems by means of integral equations. I, Quart. J. Mech. Appl. Math. 12 (1959), 232-241.

4. - On the solution of some axisymmetric boundary value problems by means of integral equations. II, Mathematika 6 (1959), 120-133.

5. - On the solution of some axisymmetric boundary value problems by means of integral equations. III, Proc. London Math. Soc. (3)10 (1960), 428-460.

6. - On the solution of some axisymmetric boundary value problems by means of integral equations. IV, Proc. Edinburgh Math. Soc (2)12 (1960/1961), 95-106.

7. - Note on the electrified spherical cap, Proc. Cambridge Philos. Soc. 55 (1959), 377-379.

8. - On the solution of some axisymmetric boundary value problems by means of integral equations. V, Quart. J. Mech. Appl. Math. 14 (1961), 101-117.

9. - Note on the electrified circular disk situated inside an earthed coaxial infinite cylinder, Proc. Cambridge Philos. Soc. 57 (1961), 623-627.

10. - The forced torsional oscillations of an elastic half-space and an elastic stratum, Proc. London Math. Soc. (3)12 (1962), 226-244. 
11. - On the solution of some axisymmetric boundary value problems by means of integral equations. VII, Proc. Edinburgh Math. Soc. (2)13 (1962), 13-23.

12. J. C. Cooke, $A$ solution of Tranter's dual integral equation problem, Quart. J. Mech. Appl. Math. 9 (1956), 103-110.

13. —, The coaxial circular disk problem, Z. Angew. Math. Mech. 38 (1958), 349-356.

14. E. T. Copson, On the problem of the electrified disc, Proc. Edinburgh Math. Soc. 8 (1947), 14-19.

15. A. E. Heins, Function-theoretic aspects of diffaction theory; Electromagnetic waves, Proceedings of a Symposium conducted by the Mathematics Research Center, Univ. of Wisconsin, Madison, Wis., 1961; pp. 99-108.

16. A. E. Heins and R. C. MacCamy, On mixed boundary-value problems for axially-symmetric potentials, J. Math. Anal. Appl. 1 (1960), 331-333.

17. - On the scattering of waves by a disk, Z. Angew. Math. Phys. 11 (1960), 249-264, 543-544.

18. - Integral representations of axially symmetric potential functions, Arch. Rational Mech. Anal. 13 (1963), 371-385.

19. P. Henrici, Zur Funktionentheorie der Wellengleichung, Comment. Math. Helv. 27 (1953), 235-293.

20. - A survey of $I . N$. Vekua's theory of elliptic partial differential equations with analytic coefficients, $Z$. Angew. Math. Phys. 8 (1957), 169-203.

21. D. S. Jones, $A$ new method for calculating scattering with particular reference to the circular disc, Comm. Pure Appl. Math. 9 (1956), 713-746.

22. E. R. Love, The electrostatic field of two equal circular co-axial conducting disks, Quart. J. Mech. Appl. Math. 2 (1949), 428-451.

23. S. D. Poisson, Journal de l'École Royale Polytechnique, Tome 12, Cahier 19 (1823), 215-248.

24. G. Temple. Whittaker's work in the integral representations of harmonic functions, Proc. Edinburgh Math. Soc. 11 (1958), 11-24.

25. A. Weinstein, Generalized axially symmetric potential theory, Bull. Amer. Math. Soc. 59 (1953), 20-38.

26. - Singular partial differential equations and their applications, Proc. Sympos. Fluid Dynamics and Applied Mathematics, Univ. of Maryland, 1961; Gordon and Breach, New York, 1962; pp. 29-49.

The University OF Michigan 